\title{
Bricolage et Improvisation, deux clefs fondamentales de la création innovante
}

\author{
Bricolage and Improvisation, two fundamental keys of innovative
} creation

\author{
Paul Bouvier-Patron ${ }^{1}$ \\ ${ }^{1}$ Laboratoire CERGAM, Université d'Aix-Marseille Toulon, France, bouvier@univ-tIn.fr
}

\begin{abstract}
RÉSUMÉ. Pour analyser le terme de bricolage dans son lien à l'innovation, nous l'envisagerons d'abord de façon formelle comme concept nouveau mais aussi dans sa perception logique et par son application actuelle. Nous l'envisagerons ensuite, comme dispositif central dans un ensemble plus vaste par son lien à l'improvisation et à l'innovation frugale pour en venir à un schéma de synthèse susceptible de contribuer à expliquer la dynamique de la création innovante. Le résultat principal est d'éclairer le rôle central du bricolage en relation à l'improvisation pour la création d'artefacts ainsi que pour innover.

ABSTRACT. In order to investigate the relationship between bricolage and innovation, we will consider it first formally as a new concept, but also in its logical perception and its current application. We will then look at it as a central mechanism in a broader context, through its link to improvisation and frugal innovation, to put forward a synthesis based on a scheme aiming to explain creative innovation dynamics. The main purpose is to shed light on the crucial role of bricolage in relation to improvisation, in order to create artefacts as well as to innovate.

MOTS-CLÉS. Abduction, bricolage, conception inventive, CtoC, économie circulaire, énaction, frugalité, improvisation, innovation environnementale, innovation grassroots.

KEYWORDS. Abduction, bricolage, circular economy, CtoC, enactment, environmental innovation, frugality, grassroots innovation, improvisation, inventive conception.
\end{abstract}

\section{Introduction}

Depuis Schumpeter [SCH 39,42], il est d'usage de distinguer innovation incrémentale (incrément par rapport à ce qui existe déjà) et innovation radicale (création détruisant ce qui existe déjà). Cette typologie rudimentaire, connaît des perfectionnements [ABE 85] par le croisement, à ce premier axe prédéfini, d'un nouvel axe concernant la dimension commerciale versus technique : ainsi, par exemple pour illustrer seulement ce nouvel axe, si Concorde est un projet technique d'ingénieur, Airbus est un projet commercial abouti. Néanmoins, la classification fondamentale binaire initiale n'a jamais été démentie et offre un tamis instrumental de référence. Toutefois, cette classification binaire s'adresse à l'offre technologique (ce qui peut en constituer une limite). Par ailleurs, toute logique classificatoire s'opère ex post : déterminer a priori si une innovation va s'avérer radicale est difficile. De plus, on insiste désormais sur les dynamiques de capacités organisationnelles et de renouvellement et/ou de création de compétences dans une démarche collective (rôle essentiel des communautés [BRO 91] ; débouchant, aujourd'hui, sur les communautés d'innovation). En outre, la demande et l'usage exercent un rôle de plus en plus important (rôle essentiel d'innovations de rupture ou Disruptive [BOW 95] marquant l'avant de l'après (ex : Internet [BOU 11]). Ces aspects rendent la dichotomie théorique initiale plus floue. En rentrant dans le coeur du réacteur de l'innovation, on s'oblige à envisager un nuancier complexe (nommé ici par simplicité Création Innovante) du côté de l'offre : découverte (résultant de la recherche scientifique); invention (activité individuelle et/ou collective de conception parfois avec les moyens du bord débouchant sur une création) ; innovation (mise en forme managériale en entreprise d'une invention par R\&D) ; conception inventive (coeur des Sciences pour l'Ingénieur SPI, cf. [PER 01]) appliquée dans un processus d'entreprise (ingénierie de recherche) ; conception technique pour les ingénieurs de production. Définissons chacun des termes retenus du spectre : 
- Découverte : ce qui est trouvé et perçu comme un accroissement important des connaissances;

- Invention : mise en cuvre technique d'une découverte;

- Innovation : invention appropriée par une entreprise ;

- Conception inventive ${ }^{1}$ : processus de transformation de l'invention en innovation réussie, avec identification du besoin ou de l'usage nouveau jusqu'à la rencontre de clients sur le(s) marché(s). Le cahier des charges fonctionnel (interne) est nécessairement ouvert pour y parvenir ;

- Conception technique de mise en œuvre productive : activité de mise en correspondance technique par rapport à un besoin identifié, sur la base d'un cahier des charges fonctionnel (interne) fermé, et le respect strict de procédures d'entreprise ainsi que des normes ISO.

Pour approfondir la question de l'innovation en la resituant de façon synthétique dans le contexte de l'entreprise, on peut renvoyer à Bouvier-Patron [BOU 11]. Par ailleurs, si le cahier des charges interne est particulièrement fondamental pour formaliser les besoins perçus et s'impose à l'entreprise, dans un dialogue entre le marketing stratégique, la $R \& D$ et les achats, il est un rouage clef en externe dans la relation client-fournisseur (pour une présentation synthétique de la question, cf. [BOU 17a]). Le cahier des charges interne est parfois une fiction au sens où l'entreprise ne s'oblige pas à formaliser de façon détaillée pour elle-même toutes les spécifications requises avec l'impartition liée dans un document unique et centralisé (ex : un cahier des charges fonctionnel puis technique par produit) mais il y a une décomposition du tout en un ensemble fragmenté dont certaines parties sont réalisées en interne et la majorité en externe (du fait du recentrage sur le métier de l'entreprise cliente comme d'ailleurs de chacun de ses fournisseurs complémentaires) dans le cadre des relations clients fournisseurs (où le pouvoir de négociation de chaque fournisseur va être déterminant pour savoir qui tient le crayon).

Insistons sur un point particulier : la distinction entre invention et innovation est essentielle pour pouvoir saisir d'emblée que l'innovation passe par un processus managérial au sein d'organisations à finalité industrielles et commerciales (à savoir, l'entreprise) quand l'invention est un processus plus évanescent en amont de l'innovation (provenant d'un individu et/ou d'un collectif) bénéficiant du cumul de connaissances antérieures mais, par une création spécifique, en agençant les idées et connaissances anciennes de façon nouvelle, en détournant les idées et/ou les connaissances ou encore basculant dans l'émergence de connaissances nouvelles.

Nous verrons que le bricolage, intuitivement très présent au stade de l'invention, est, également, particulièrement mobilisable au stade de l'innovation et de la conception.

Pour l'instant, indiquons que le mythe managérial moderne de l'entreprise serait de parvenir à planifier l'innovation. Or, ceci s'avère particulièrement délicat même pour les entreprises adoptant les meilleures dispositions (décentralisation et latéralisation organisationnelle, incitation à la création par du temps de travail libéré, encouragements et aides aux projets). Par ailleurs, pour toute entreprise, innover impose, pour le moins, des compétences et des dépenses en R\&D minimales, pour disposer de capacités internes d'absorption [COH 90] mais aussi désormais un fonctionnement en innovation ouverte [CHE 03] compte tenu du besoin d'interactions collectives et de mutualisations de par la nécessite pour chacun de se spécialiser sur son métier tout en ayant besoin de complémentarités. Cependant, tout ce qui favorise l'innovation n'implique pas en sortie une innovation (et, si oui, non nécessairement d'ailleurs valorisable économiquement et encore moins économiquement rentable). Le processus innovant est long, coûteux et incertain (ce qui en démultiplie le coût et justifie, d'une certaine manière, le prix pratiqué à la vente) : ceci implique pourquoi le marketing stratégique et le marketing de l'innovation occupent une place de plus en plus prépondérante dans l'entreprise.

\footnotetext{
${ }^{1}$ La conception inventive renvoie principalement à la méthode TRIZ [ALT 88] ou théorie de la résolution des problèmes inventifs particulièrement mobilisée par les SPI. En SHS, le Design Thinking [BRO 08], s'ancrant dans les travaux de Simon [SIM 69], est plutôt mis en avant.

(C) 2021 ISTE OpenScience - Published by ISTE Ltd. London, UK - openscience.fr 
Si l'on se recentre sur la question de la création au sens large (regroupant les phases, sans lecture linéaire nécessaire entre elles, de découverte, d'invention, d'innovation, de conception inventive), le bricolage est très présent du fait du besoin de tâtonnement nécessaire. Pour bien discriminer logiquement les phases nécessaires pour innover, on peut s'appuyer sur le couple création/conception, la création étant en amont de la conception (jusqu'à la conception technique une fois le processus maîtrisé pour la mise en phase industrielle et commerciale).

Le bricolage renvoie à une pratique transversale et propre à illustrer l'importance du faire dans la résolution de problèmes. C'est aussi l'illustration concrète et naturelle de l'idée que la main est le prolongement indispensable du cerveau humain.

De façon basique, le bricolage désigne une pratique de création, de réparation créative et/ou de détournements d'usage, essentiellement à l'aide des moyens du bord (outils, méthodes, espaces, matériaux) et sans visée commerciale a priori. Le bricolage est une pratique favorisant, au-delà de l'expérience déjà acquise, grâce à l'apprentissage mais aussi l'intuition, l'expérimentation et l'interactivité, la résolution de problèmes plus ou moins complexes jusqu'à l'élaboration d'objets au moyen d'outils (rudimentaires en général mais pouvant être sophistiqués) par le développement d'un savoir-faire adaptatif et ouvert mobilisant une forte part de créativité (et/ou de co-créativité dans un cadre collaboratif) où donc, comme pour toute activité innovante, quelque chose de nouveau, ici par la pratique, est incorporé à l'existant (pour une création, une modification, une transformation, une réparation fonctionnelle ou un détournement d'usage) soit marginalement, soit structurellement.

Pour analyser le terme de bricolage dans son lien à l'innovation, nous l'envisagerons (1.) de façon formelle (1.1) en tant que concept nouveau puis (1.2) dans sa perception logique et, enfin, (1.3) par son application actuelle ; mais aussi (2.) comme dispositif central dans un ensemble plus vaste par son lien (2.1) à l'improvisation et (2.2) à l'innovation frugale ; (2.3) pour en venir enfin à un schéma de synthèse susceptible de contribuer à expliquer la dynamique de la création innovante.

\section{Le bricolage comme concept}

Il convient d'appréhender le bricolage (1.1) en tant que concept nouveau puis (1.2) dans sa perception logique et (1.3) par son application actuelle.

\subsection{Le bricolage : concept nouveau, pratique ancienne}

Le bricolage est une pratique fondée sur l'apprentissage, l'expérience, l'intuition, l'expérimentation par essai-erreur (y compris via le hasard par sérendipité : trouver en ne cherchant pas ou en ne cherchant pas la chose trouvée ; ce qui n'est pas sans incidence au-delà sur la difficulté de pouvoir planifier l'innovation) mais aussi l'interactivité. C'est une démarche créative adaptative à forte implication individuelle (pouvant s'inscrire dans un cadre collaboratif) passant par l'acquisition d'astuces dans une base de connaissances, généralement tacites, constituée par accumulation collective transmise de bouches à oreilles et/ou par le biais associatif et/ou encore par l'accès, via internet, à un corpus numérisé (figé ou évolutif mais accessible et parfois interactif) s'appuyant sur le don et le contre don (au sens du fondateur de l'anthropologie M.Mauss : Essai sur le Don en 1923), à savoir une forme d'échange effectif mais via une transaction à valeur symbolique complexe d'ordre sociétal), la réciprocité d'échanges (équivalence) ou la mise à disposition unilatérale gratuite. Pourtant très ancien déjà, le bricolage est un concept nouveau à explorer et à (re)placer à son réel niveau d'importance.

Le bricolage a été mis en évidence en Sciences Humaines et Sociales (SHS) par [LEV 62] comme pratique clef au sein des sociétés primitives, lesquelles mobilisent astucieusement les ressources naturelles à leur disposition pour satisfaire au mieux leurs besoins. Ce concept trouve aujourd'hui un écho renouvelé par l'activité des Makers [AND 12], mobilisant les nouvelles technologies (Information\&Communication ou TIC et Numérique ou TN), au sein de communautés de pratique 
[BRO 91], par la création/co-création (puis la conception/co-conception) à travers la réalisation de nouveaux objets, voire la réparation ou encore le détournement d'usage des objets, et ce de façon individuelle (DIY : Do It Yourself) ou collective (DIWO : Do It With Others) dans une optique fermée propriétaire (avec une perspective de valorisation commerciale) versus ouverte [RAY 99] en Open Source (pour développer des applications ouvertes de partage et/ou à finalités commerciales).

\subsection{Le bricolage, affaire de logique?}

Classiquement (depuis John Stuart Mill en 1843 avec son Système de logique inductive et déductive), les économistes opèrent la distinction entre logique inductive et déductive. La pensée scientifique théorique abstraite et non expérimentale mobilise généralement la logique déductive. Au contraire, certaines sciences, essentiellement empiriques (comme les sciences naturelles comme la zoologie où l'on dénombre et classifient les espèces), mobilisent plutôt la logique inductive. D'autres sciences comme la physique combinent logique inductive et déductive. Cependant, cette caractérisation n'est pas exclusive et Aristote déjà spécifiait une forme d'induction particulière (nommée rétroduction) reprise par Peirce (cf. collected post-mortem papers [PEI] et/ou [PSI 11]), sous le terme d'abduction.

L'empire logique est donc plus complexe qu'il n'y semble et les mathématiciens, par exemple, revendiquent assez fortement l'importance de l'intuition permettant d'inférer un résultat en se saisissant d'une singularité non encore démontrée pour, ensuite, procéder formellement à un énoncé démontré. Cette possibilité en mathématiques liée à l'abstraction peut aisément être généralisée au raisonnement scientifique produisant un discours sur une réalité extérieure, cette réalité offrant naturellement des singularités et permettant l'émergence de faits surprenants (The surprising fact $C$ is observed, cf. Pragmtism and Abduction,1903 (5.189) [PEI]).

Ce sont ces faits surprenants qui, par la perturbation qu'ils induisent dans le raisonnement, obligent à produire une explication nouvelle, à savoir non existante et/ou non étalonnée dans la base de connaissance prévalante.

Ainsi, d'un fait surprenant, l'abduction permet une remontée en arrière (d'où la rétroduction) pour formuler une hypothèse nouvelle acceptable expliquant ce qui s'est passé lors de l'observation de la surprise. A ce stade, l'abduction ne donne que des hypothèses possibles. La sélection de l'hypothèse donnant l'explication du fait dépend de plusieurs critères : le pouvoir prédictif de l'énoncé liée à l'hypothèse formulée ; la vérifiabilité expérimentale (permettant la reproduction du fait) à partir d'un dispositif associé à l'hypothèse ; le choix de l'hypothèse assurant la plus grande simplicité explicative car le plus simple est le plus facile à tester (l'hypothèse s'avérant fausse, il est alors facile de l'éliminer pour partir alors sur une hypothèse plus compliquée) ; l'hypothèse la plus ouverte car ne fermant pas le raisonnement de façon limitative.

Chez Peirce, l'enjeu est d'articuler les trois formes déduction, induction, abduction qui sont complémentaires. L'abduction constitue un aperçu créatif (A Creative Insight) pour résoudre un problème surprenant. L'abduction constitue un pari interprétatif. Au total, l'abduction est cette inférence particulière qui, à partir d'un fait surprenant invite une induction spécifique permettant d'élaborer une hypothèse explicative non formulée dans la base de connaissances existante. Le fait surprenant est la base du processus (de découverte, de création ou d'innovation) par la sérendipité.

Le fait surprenant est récurrent dans la pratique du bricolage et permet d'apporter ensuite la bonne réponse au problème posé. La pratique du bricolage induit un recours naturel à l'abduction : une réponse pragmatique est apportée à des faits surprenants (séquentiels) jusqu'au tâtonnement vers un compromis trouvé d'une solution globale satisfaisante [SIM 76,78]. Pour résumer et étendre le propos sur l'abduction qui intéresse bien des domaines de la vie, retenons par exemple une formulation concise quoique abstraite [CHE 14] : "l'abduction, qui recherche des causes probables à des effets certains, aboutit à des énoncés plausibles". 


\subsection{Le bricolage, application actuelle du concept}

Le bricolage s'inscrit dans un spectre large dans un nuancier de gradation des moyens technologiques mobilisés à partir d'une base qui est l'utilisation des moyens du bord. Il s'agit d'une logique individuelle et/ou collective plus ou moins formalisée et organisée de partage, d'échange, d'apprentissage et de réalisation effective. La finalité commerciale n'est pas à exclure lorsque l'art du bricolage est utilisé dans les organisations à but lucratif ; de façon indirecte par les communautés d'innovation non exclusivement liées aux entreprises ou de façon directe lorsque les communautés d'innovations fonctionnent comme des appendices au service d'entreprise(s).

Les communautés d'innovation associent, autour d'un projet ne pouvant être utilement ou efficacement conduit (au sein d'une entreprise ou dans la sphère inter-organisationnelle par la seule coordination du réseau client-fournisseurs), des savoirs, des savoirs faire et des compétences interdisciplinaires complémentaires, avec un ancrage plus ou moins technologique, en se réunissant dans un lieu physique (Makerspace) ; nommé plateforme d'innovation si dédié à un projet spécifique mobilisant généralement un lieu-outil FabLab [GER 05, BOU 15a] et d'autres dispositifs technologiques ou organisationnels (espace de Co-Working, incubateur pour jeunes pousses, accélérateur accompagnant la croissance d'une StartUp mature, Show Room avec espace d'essai pour permettre des tests d'usage). Le lieu peut exister, virtuellement, de par une communauté virtuelle d'innovation reliant, par Internet, de façon interactive et dynamique, des individus ou des collectifs passionnés autour d'une idée, d'un projet ou d'un centre d'intérêt attractif (ex : marque), pour engendrer une intelligence collective et/ou de passer à une réalisation commune.

Le bricolage est essentiel dans le processus de création ou de co-création d'objets pour en explorer et tester le système de contraintes et voire même parvenir à élaborer une maquette évolutive (éventuellement numérique) selon la complexité à considérer ou, dans tous les cas, un premier prototype plus ou moins fonctionnel. Le bricolage mobilise les ressources disponibles sans objectif de rentabilité (immédiate) mais avec l'idée d'expérimenter des solutions et de cheminer pour rechercher un compromis acceptable (Satisficing : [SIM 76, 78]), d'après les contraintes révélées (par une communauté de référence ou au-delà). Ce qui se traduit en termes d'objectifs possibles, de fonctionnalités à envisager, d'usages potentiels, de coûts, de moyens, de résultats attendus ou bien pour s'ouvrir à des solutions ou résultats inattendus (ex : sérendipité) en invitant au croisement de champs disciplinaires (SHS/Sciences/SPI) et en articulant tous les niveaux concernés (conception, production, diffusion, usage) ainsi que potentiellement l'ensemble des parties prenantes (actionnaires, salariés, clients, fournisseurs, associations concernées par l'impact positif/négatif de l'entreprise).

A l'issue de cette première partie permettant de situer l'importance conceptuelle du bricolage, nous pouvons en montrer l'importance, de façon extensive mais non exhaustive, comme dispositif dans un ensemble plus large.

\section{Le bricolage comme dispositif clef}

Le bricolage s'inscrit comme un dispositif central dans un ensemble plus vaste, par son lien (2.1) à l'improvisation et (2.2) à l'innovation frugale. Ce qui permet d'établir (2.3) un schéma de synthèse ouvrant des perspectives de recherche théoriques et appliquées sur la dynamique de création innovante.

\subsection{Bricolage et Improvisation}

Il conviendra de distinguer les concepts de bricolage (où la dimension de rareté est essentielle en mobilisant, en première instance, les moyens du bord et sans contrainte temporelle imposée -sauf, de façon pratique lors d'une phase le nécessitant ou encore de par un danger imminent-) et celui d'improvisation (où le temps d'exécution d'une solution trouvée -par émergence spontanée, éduquée 
et/ou d'entrainement-, pour faire face à une situation nouvelle, est essentiel dans un contexte concurrentiel et de performance). Les deux concepts sont distincts et complémentaires.

Dans l'improvisation, la dimension temporelle s'impose pour parer au plus vite et de façon adaptée, à tout évènement imprévu par rapport à l'organisation habituelle de l'activité prescrite ou réalisée effectivement et sans que ne soit présente, pour l'essentiel, la contrainte de rareté. La littérature managériale est plutôt orientée à ce jour sur ce dernier concept d'improvisation ([WEI 93], [CUN 14]).

Weick [WEI 93], pour stipuler l'influence managériale en situation d'incertitude avec des tâches complexes à accomplir, mobilise le concept d'énaction (anglicisme littéral : Enaction) pour signifier qu'une mise en place organisationnelle particulière s'opère au moment où les individus, individuellement et/ou collectivement, agissent par initiative de façon interactive avec leur environnement situé ; ce qui n'est possible que par une processus, orienté vers l'action, de construction de sens (Sensemaking) commençant par l'identification d'éléments inattendus (ou faits surprenants chez Peirce) et contextualisés afin d'obtenir une représentation commune (une divergence d'appréciation dans l'urgence et face à l'imprévu ayant toujours des conséquences désastreuses); et ce, dans une dynamique de changement permanent où la coordination en continu prime grâce à la mobilisation de règles existantes adaptées et/ou à la création de nouvelles règles et des rôles bien distribués dans le collectif. C'est là le socle pour saisir l'importance de l'improvisation.

Au-delà de Weick, et pour montrer le caractère générique du problème posé, [MOO 98ab] illustrent parfaitement l'improvisation, en citant d'autres auteurs spécialisés, à travers la question de l'évitement de la catastrophe : par l'exemple, celle de la mission Apollo XIII de la NASA où diverger (des routines induites de par les procédures admises et planifiées), dans l'urgence, a été le seul recours -en mobilisant toutefois un haut niveau de professionnalisme à capacité adaptative (tout en s'aidant du bricolage fautil le souligner)- dont le résultat a permis le retour sur terre de l'équipage promis pourtant à une mort certaine (envisageable dès le lancement de la fusée de par un incident survenu soudainement et affectant un élément vital de la structure).

$\mathrm{Si}$, a priori, l'on pourrait arbitrairement penser opposer bricolage (d'amateur, dénotant une forme d'immaturité) et improvisation (du professionnel disposant d'une grande expertise), [BAK 03] montrent bien, au stade professionnel, qu'il y a une complémentarité au sens où la compétence acquise par le bricolage donne l'aptitude à improviser.

L'ingénieur en entreprise, dans une logique standard, fonctionne a priori avec un accès à des ressources répertoriées mais aussi respecte des procédures ou des règles selon des contraintes temporelles et un échéancier prédéfini. Le même ingénieur qui doit trouver une solution à un problème inattendu, alors qu'il est pourtant placé au départ dans une logique standard, va devoir improviser. Le même ingénieur, participant à un projet innovant, à un moment ou un autre (ou même en continuité), va devoir, seul ou collectivement, sortir de la routine (parfois de façon radicale) et improviser (sous contrainte temporelle) mais aussi bricoler (et en cherchant parfois lui-même les ressources nécessaires et/ou en les récupérant) pour inventer, à partir de rien ou par détournement, un dispositif qui lui est nécessaire il doit mobiliser des ressources et moyens hétérogènes et non nécessairement répertoriés, opérer par amalgame ou dosage intuitif, agencer, configurer pour ébaucher une solution viable. On peut donc parfaitement discriminer l'improvisation comme une urgence impliquant une action dérogatoire dans un cadre donné et le bricolage par l'absence d'urgence mais la genèse créative à partir de rien ou presque rien (avec les moyens du bord, avec les ressources localement disponibles et, souvent, par la récupération) pour trouver une solution acceptable sans besoin d'une codification préalable [LEV 62], [CUN 14], [HAL 12].

La littérature managériale, sur les questions du bricolage et de l'improvisation, est parfois complexe à suivre sur le plan diachronique de par l'absence d'homogénéité et de stabilité conceptuelle. En sélectionnant correctement les auteurs, l'essentiel peut être mis en évidence : 
- certains [BAK 03] distinguent bien improvisation et bricolage (sans cependant vraiment sortir de la vision réductrice selon laquelle le bricolage ne serait qu'une étape dans le processus d'improvisation ${ }^{2}$ ) ; en outre, [BAK 03], en s'appuyant sur [MOO 98b], proposent de voir l'improvisation (mais aussi donc, implicitement, le bricolage) non comme une opération purement tactique (opportuniste) mais plutôt stratégique (essentielle voire vitale) dans la création de nouveaux produits ;

- d'autres [NOH 94] proposent d'emblée des éclairages pertinents sur le bricolage : "Bricoleurs play with possibilities and use available resources to find workable solutions" ... "The activities of bricolage occur when people explore existing resources in novel ways, not necessarily under time pressure as in the case of improvisation" ;

- enfin, s'appuyant sur [MOO 98a], [CUN 14] réaffirment clairement ce qu'est l'improvisation : "Improvisational innovation occurs when there is time pressure" ... tout en parvenant à faire face sans disposer pour autant de ce qu'il aurait fallu de façon adéquate en situation optimisée (et ce, sans aucun lien premier avec une rareté sur la ressource) ; avec, en outre, la précision suivante [CUN 14], en s'appuyant sur [MIN 01] : "Improvisation is normally not favored in product innovation because it entails significant risks... We suggest that, under normal conditions, people would prefer not to improvise given the risks involved" ${ }^{3}$. La question du choix préférentiel mentionné est cependant relative car les circonstances jouent un rôle déterminant ${ }^{4}$ en la matière.

Pour l'art de l'improvisation, le défi est d'anticiper l'adaptation requise pour parvenir à créer (ou cocréer) ou seulement rétablir une situation conforme (évolutive ou non). L'improvisation peut, éventuellement, bénéficier d'une phase préalable active d'entrainement sur la base des consignes, règles, guides ou scénarios (allant du plus au moins vraisemblable), selon une hypothèse de couverture d'un spectre vraisemblable des possibles (sans toutefois jamais pouvoir être exhaustif). Or, le cas critique, dans le cadre d'une routine organisationnelle, est justement la survenue d'une situation inattendue et dérogeant à ce qui a été prévu, formalisé et/ou planifié : ce cas critique implique, pour y répondre, un art d'improviser (reposant sur l'entrainement, une pratique, une expérience et un savoirfaire) et peut mobiliser, au besoin, l'art du bricolage.

\footnotetext{
${ }^{2}$ Cependant, les deux concepts sont orthogonaux au sens mathématique du terme dans la mesure où l'on peut bricoler sans nécessairement improviser (ex : invention), improviser sans nécessairement bricoler (ex : faire face à une urgence pour palier une défaillance dans la routine industrielle) bricoler et improviser (ex : conception inventive) ou encore n'i l'un ni l'autre (ex : produire par un mode programmé et/ou par le fonctionnement organisationnel imposé de type Taylorien-Fordien)... Quoique, même dans ce dernier cas, une discipline relativement récente comme l'ergonomie (résultant de l'étude de l'homme en situation limite durant la 2ème Guerre Mondiale) -avec une école française s'opposant à l'ergonomie anglo-saxonne en ce que cette dernière tendait plutôt à adapter l'homme à la machine quand la première entend adapter la machine à l'homme, mais pour laisser place désormais à une vision interactive en tenant compte de l'ambiance (bruit, odeur, humidité, chaleur-, de la pénibilité et de l'exposition aux risques)- montre, notamment, l'importance, individuellement et collectivement, de la représentation d'une situation de travail et du différentiel entre le prescrit (ex : prescrit par la planification du bureau des temps et méthodes dans le schéma Taylorien) et le réel, à savoir les pratiques effectives des équipes ou d'individus, sur le terrain, pouvant mettre en oeuvre des astuces ou solutions amendant utilement la planification initiale pour permettre la réalisation effective, parfois même en adaptant l'outillage ou l'organisation.

${ }^{3}$ Notons que l'aversion au risque est une préoccupation centrale de la littérature académique en économie et en management mais que, si cet aspect n'est certes pas à négliger et rentre bien dans le champ réel des possibles, la prise de risque est nécessaire et inévitable (maitrisée si possible, à des degrés divers) dans les activités comme le travail en milieu confiné (ex : salle blanche), celles à haut risque (ex : scaphandrier sous la mer ou spationaute de par les contraintes techniques et de milieu), celles en lien direct avec des éléments pathogènes (ex : laboratoires, hôpitaux), celles de maintenance (ex : chimie, pétrochimie, nucléaire); et/ou celles souvent extrêmes et périlleuses (ex : opérations de sauvetage en cas d'incendie, de catastrophes industrielles ou naturelles). Plus encore, la prise de décision en incertitude [KNI 21], aujourd'hui décentralisée du chef d'entreprise à des personnels hiérarchiquement subalternes (autre débat que celui de la responsabilité des dirigeants salariés ou Salaried Managers), est d'ailleurs, pour cet auteur, le fondement même de la vie économique.

${ }^{4}$ En cas d'épidémie (et, a fortiori, de pandémie), par exemple, qui implique une réorganisation adaptative des personnels, des compétences, des soins, des lieux, des moyens, il faut mobiliser précisément et nécessairement improvisation (ex : transférer du matériel du bloc opératoire pour un autre usage ; adapter une salle de réveil post-opératoire en unité opérationnelle de réanimation confinée) et bricolage (ex : façonner soi-même un masque efficace). L'impression 3D pour fabriquer des objets existants manquants (ex : embouts de tubage) peut relever du bricolage technologique s'il y a tâtonnement, partant de l'existant, pour concevoir le modèle numérique nécessaire, ce qui peut conduire par là même aussi à améliorer l'objet ou, voire, à plus radical encore (cf. infra, Schéma1). (C) 2021 ISTE OpenScience - Published by ISTE Ltd. London, UK - openscience.fr

Page $\mid 7$
} 
Quant à lui, l'art du bricolage remet en cause fondamentalement le concept d'efficience managériale (mise en relation des moyens mobilisés pour parvenir au résultat ; déjà opposé au concept d'efficacité où la fin justifie les moyens pour parvenir au résultat). En effet, l'art du bricolage permet d'obtenir un résultat en mobilisant souvent très peu de moyens ou des moyens de fortune, inattendus ou surprenants, tout en accédant bien au résultat. On peut donc considérer que le bricolage participe fortement à l'innovation organisationnelle et peut même en être un déclencheur.

Les pratiques de bricolage et/ou d'improvisation sont compatibles et peuvent s'exercer à titre individuel ou collectif, dans un espace fermé ou ouvert, privatif ou associatif, avec ou sans but lucratif. L'art du bricolage et l'art de l'improvisation (réunis ou séparés) concourent à la création et à la cocréation puis la conception et la co-conception inventive, par la pratique fondée sur l'expérience, le savoir-faire, l'intuition, l'expérimentation, l'interactivité, les échanges d'expérience et les mises en commun tout en actant l'importance cruciale dans l'innovation de l'expérimentation et du hasard (sérendipité).

\subsection{Bricolage et Innovation Frugale}

L'art du bricolage et l'art de l'improvisation, utiles à la créativité (et à la co-créativité dans le cadre collectif), peuvent être favorisés par les moyens technologiques ( $F a b L a b$ en tant que couteau Suisse permettant de faire presque tout [GER 05]) et/ou l'organisation (MakerSpace) mais aussi par la méthode présidant à la pratique (sophistiquée High Tech ou bien par la frugalité).

Le débat sophistication versus frugalité est essentiel dans un contexte de rareté croissante des ressources et de la rationalisation des coûts mais aussi, potentiellement, de prise en compte des contraintes environnementales.

La frugalité elle-même mérite de s'y arrêter pour en préciser le champ, ce vocable ne renvoyant pas au même contenu pour tout un chacun. La frugalité doit être clairement dissociée de la question de l'inversion d'une part mais aussi de la circularité d'autre part.

Il y a inversion du flux habituel (perçu à travers la courbe de cycle de vie du produit [VER 66]) si l'exportation est originaire d'un pays de type BRIC et le destinataire un pays occidental. Toutefois, cas de délocalisation, on ne peut parler d'inversion si le produit est ré-exporté vers l'occident (tout en bénéficiant de coûts sociaux et environnementaux faibles ou inexistants). Deux modèles économiques s'opposent alors, le Low Cost (destiné principalement à l'exportation ou la ré-exportation) et le $B O P$ (Bottom of the Pyramid: [PRA 05]) où il s'agit de servir d'abord la base de la population (tous mais d'abord la masse) : logique d'inclusivité. Le BOP repose sur la frugalité d'origine indienne [BOU 17b] et se décline en deux modalités principales : Gandhian (plus avec moins pour beaucoup) ; Jugaad (plus avec moins pour beaucoup mais de bonne qualité et désirable/attractif).

La démarche frugale d'innovation (innovation frugale) mobilise le bricolage au stade de la création (et co-création) et de la conception (et co-conception) d'objet(s) mais invite à une logique aboutie de réalisation d'un produit mobilisant le moins de ressources possibles immédiatement disponibles (et généralement rares). Par opposition, la sophistication peut recourir à un bricolage technologique ([AND 12], [GER 05]) au stade de la création (co-création) et de la conception (co-conception) au stade proto-industriel.

L'innovation frugale consiste à faire ce qui existe déjà ou ce qui n'existe pas encore avec moins, moins cher et mieux sans mobiliser la sophistication tout en proposant, pour l'usage, au moins autant de fonctionnalités (souvent de nouvelles) ainsi qu'une qualité de conception et de réalisation produit mais aussi pertinence (adéquation aux besoins locaux réels : produit adapté, abordable, robuste, réparable), effectivité (facilité d'utilisation), évolutivité potentielle. 
Pour autant, il n'y a pas économie circulaire au sens où les déchets engendrés par ailleurs n'ont pas été nécessairement prévus pour le réemploi comme intrants. Or, l'économie circulaire implique de penser la production d'objets pour leur réemploi [GEY 04].

Pour autant, même en cas d'économie circulaire, celle-ci n'est pas en soi environnementale (à savoir, permettant de réduire ou d'éliminer l'impact environnemental). Le CtoC (Cradle to Cradle du berceau au berceau [BRA 02], qui s'oppose à la dynamique initiée par la révolution industrielle du Cradle to Grave (du berceau à la tombe par le cycle extraction/ production/ consommation/ déchets ; avec, dans le meilleur des cas, recyclage partiel par un raisonnement de traitement ex post ou End of Pipe -contrairement à une intégration du cycle de vie dès la conception même- cf. [HAR 84]), peut donc être envisagé, ou pas, comme vertueux sur le plan environnemental selon qu'il permet (ou pas) d'élaborer des produits respectueux de l'environnement avec des intrants écologiques et ré-employables indéfiniment. Les conséquences environnementales s'analysent tout au long du cycle de vie, tant au niveau de la conception, la réalisation, la vente, l'usage et la mise au rebut. L'éco-conception bien conduite doit justement anticiper les conséquences environnementales et peut s'inspirer de la nature [BEN 97].

C'est le propos de l'innovation Grassroots (décentralisée, collective, respectueuse de l'environnement [SEY 07]), latéralisée car reposant sur des compétences réparties entre pairs et induisant bon sens, bricolage et/ou voire bricolage technologique mais aussi débrouillardise individuelle/collective (Système D), en prenant d'emblée en compte l'environnement à tous les stades de vie du produit sans, pour ce faire, mobiliser une sophistication inutile ou excessive (renvoyant à une sobriété technologique ou à un compromis technologique satisfaisant : Satisficing [SIM 76,78]). On peut accéder alors au concept d'Innovation Frugale Environnementale (ou l'IFE [BOU 17b,20]).

L'innovation Grassroots est une forme de frugalité qui, mobilise fortement le bricolage, en l'associant au réemploi de ressources disponibles (résultant de déchets constitués par des objets en fin de vie pour différentes raisons : défectuosité, fonction non-réparable, casse, pièce détachée introuvable).

Ces déchets seraient-ils le fait d'une obsolescence massive?

Le terme d'obsolescence programmée est attesté au moins depuis 1932 (à travers la publication aux USA d'un opuscule intitulé Ending the Depression through Planned Obsolescence de B.London). C'est cependant avec le Designer industriel B. Stevens (Brooks Stevens Design Associates : BSDA), que nait, dans les années 50, le concept d'obsolescence esthétique, à savoir produire des biens attractifs mais pouvant être rendus obsolètes rapidement en créant un désir de renouvellement et de changement. L'obsolescence est provoquée en inculquant à l'acheteur le double désir d'un toujours plus récent pour s'en défaire un peu plus tôt que nécessaire. A.Sloan, dirigeant (1923 à 1956) de General Motors, outre la mise en place de la forme divisionnelle -dont il est concepteur et maître d'œuvre (Chandler [CHA 62] la théorisant ensuite)-, est un précurseur aussi en promouvant, dès les années 30, sur la base du couple châssis-moteur, une gamme de véhicules par la carrosserie (en jouant sur la forme, la couleur, les accessoires) renouvelée annuellement par stimulation publicitaire. L'obsolescence programmée par la mode et/ou le Design est un fait.

Le débat contemporain sur l'obsolescence programmée s'oriente sur une théorie du complot (pour approfondir cette question au niveau sociétal, cf. [CHE 14]) avec suspicion de planification de défectuosités, de fragilités, de vie écourtée ; et ce donc, indépendamment des arbitrages nécessaires entre puissance-efficacité-coût d'un produit vendu (un produit est optimisé sur des fonctionnalités et/ou des capacités à un moment donné mais sera dépassé par l'évolution technologique). Un produit devrait toutefois pouvoir être réparable. Or, c'est peu le cas. L'essentiel des produits proposés, aujourd'hui encore, sont jetables (éventuellement alors recyclables mais peu conçus pour leur réemploi circulaire). 
Le bricolage peut être mobilisé pour réparer, en tout ou partie, un objet et/ou se servir des déchets en extrayant les pièces détachées nécessaires pour un réassemblage ou encore assembler différemment pour réaliser autre chose. Les déchèteries sont essentielles dans la mise à disposition des matériaux de réemploi : les stocks déclassés alimentent des ressourceries, improvisées localement ou organisées par filière et par volume (à grande échelle et par maillage dans les Pays Développés). Dans les pays les moins avancés (PMA) ou les pays pauvres sans préoccupations sanitaires et/ou environnementales, il semble encore difficile d'élaborer des filières de recyclage respectueuses de la santé des populations et de l'environnement. Commencer à instaurer un cercle vertueux ne résout pourtant pas la question de la résorption du stock de déchets amoncelés, au niveau global, sur terre, sous terre ou dans les mers.

\subsection{Schéma synthétique d'analyse}

$\mathrm{Au}$ terme de cette analyse, pour appréhender les axes pertinents relatifs au bricolage innovant, on peut en retenir au moins quatre principaux :

- l'existence actuelle de la ressource pour discriminer ce qui existe déjà de ce qui est nouveau ;

- la contrainte temporelle forte ou faible impliquant un degré d'urgence ;

- la rareté signifiant la disponibilité intrinsèque effective de la ressource ;

- la créativité selon le niveau d'effort créatif dont l'appréciation est nécessairement qualitative.

Les deux premiers axes n'appellent pas de commentaires particuliers et sont intuitivement compréhensibles, d'autant qu'une quantification peut être réalisée.

La rareté peut en fait être considérée comme un paramètre ajustable (selon la ressource) pouvant même admettre une valeur binaire $(0,1)$ pour signifier un changement de paradigme du fait d'une modification soudaine des conditions économiques ou géopolitiques changeant du tout au tout la disponibilité de cette ressource (ex : crise, guerre, catastrophe industrielle, catastrophe naturelle, etc.).

La créativité s'exprime très notablement dans certains secteurs d'activité (comme l'artisanat, le luxe, la mode, le développement produit, la recherche appliquée -terme creux où l'on peut classer la conception inventive-, la recherche fondamentale ou l'art), et en relation aux usages, mais toujours à un niveau d'intensité qui va dépendre des dynamiques individuelles et collectives ainsi que de l'importance des enjeux (d'où la couronne suspensive en pointillé autour de l'axe créativité sur le Schéma 1). La nature intrinsèquement qualitative de cette variable est patente. Le croisement des deux premiers axes peut être considéré robuste et pertinent (sauf donc situation économique extrême engendrant une rareté absolue et sans, par ailleurs, préjuger de la créativité). Ce croisement permet d'établir une matrice typologique (Tableau 1) des types de bricolage et d'improvisation.

\section{Typologie Élémentaire}

\begin{tabular}{|c|c|c|}
\hline Temps & $\begin{array}{c}\text { Contrainte } \\
\text { Temporelle } \\
\text { Faible }\end{array}$ & $\begin{array}{c}\text { Contrainte } \\
\text { Temporelle } \\
\text { Forte }\end{array}$ \\
\hline $\begin{array}{c}\text { Ressources } \\
\text { Existantes }\end{array}$ & $\begin{array}{c}\text { Bricolage 1 } \\
\text { (Incrémental) }\end{array}$ & $\begin{array}{c}\text { Improvisation 1 } \\
\text { (Incrémental) }\end{array}$ \\
\hline $\begin{array}{c}\text { Ressources } \\
\text { Nouvelles }\end{array}$ & $\begin{array}{c}\text { Bricolage 2 } \\
\text { Technologique } \\
\text { (Radical) }\end{array}$ & $\begin{array}{c}\text { Improvisation 2 } \\
\text { (Radical) }\end{array}$ \\
\hline
\end{tabular}

Tableau 1. Typologie Élémentaire (source auteur) 
Partant du tableau1, par projection et superposition des informations qualitatives listées au cours du développement explicatif (cf. supra), on obtient une cartographie conceptuelle (Schéma1) permettant de visualiser les effets d'entrainement du bricolage et de l'improvisation ainsi que de monter les points saillants de la dynamique de création innovante.

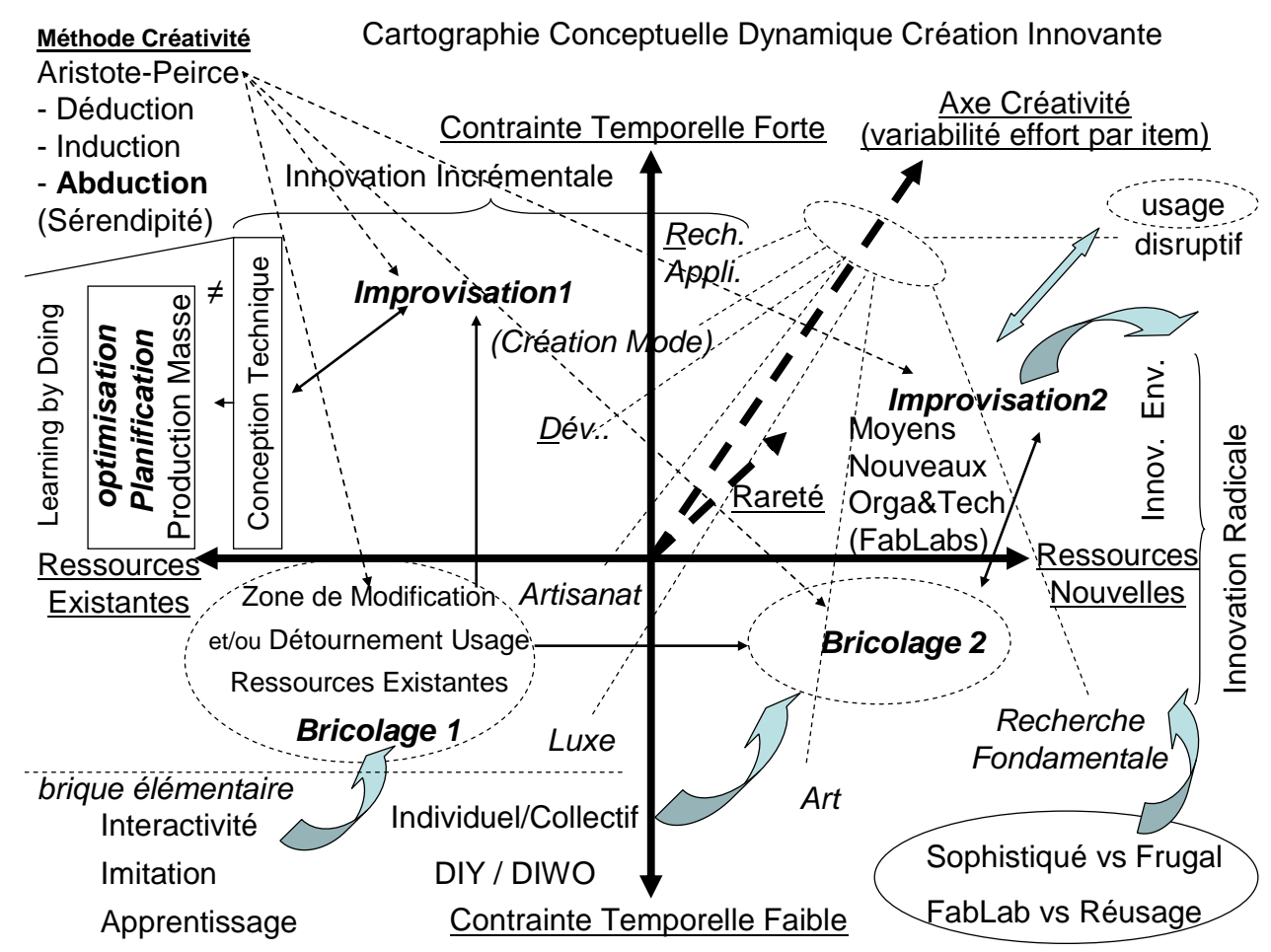

Schéma 1. Schéma de Synthèse (source auteur)

\section{Conclusion}

Que ce soit dans une démarche frugale (donc, en première instance, sobre en technologie ou, plus exactement, mobilisant un niveau ou degré technologique satisfaisant au sens de Simon [SIM 76,78]) au sein d'ateliers rudimentaires ou dans des lieux plus sophistiqués mobilisant les technologies numériques comme l'impression ${ }^{5}$ 3D (FabLab ou, plus largement, Makerspace), le bricolage (basique et/ou technologique) est essentiel autant dans la création (et la co-création) d'œuvre intellectuelle et/ou artistique que dans la conception (co-conception) d'artefacts. Le bricolage est une pratique nécessaire indispensable, depuis les âges primitifs de la civilisation jusqu'aux Makers modernes, pour rechercher et trouver la ou les solutions aux problèmes survenant dans l'élaboration de projets et/ou d'objets dans un cadre individuel ou collectif. Le bricolage est indissociable de l'expérimentation au cours du cheminement d'une pratique et fait appel à l'intuition, la créativité, l'apprentissage, l'expérience acquise ; l'interactivité, la mobilisation de trucs et astuces pour élaborer, réparer, détourner des objets (déjà existants ou inventés), généralement donc avec les moyens du bord mais aussi par des moyens technologiques sophistiqués. Le bricolage est un concept distinct mais lié à l'improvisation. L'art du bricolage et/ou de l'improvisation existe dans toute entreprise : de l'entreprise individuelle à la très grande entreprise en passant par la StartUp. Comme nous avons essayé de le montrer, l'art du bricolage et/ou de l'improvisation sont deux ressorts clefs de la dynamique de création innovante. Le schéma de synthèse proposé est susceptible d'expliquer mais aussi d'ouvrir des perspectives nouvelles de recherche concernant la création innovante.

\footnotetext{
${ }^{5}$ L'impression tri-dimensionnelle (3D) relève d'une méthode de fabrication Additive (Additive Manufacturing) -ajout de matière par couche-, par opposition à la méthode de fabrication habituelle Soustractive connue dans l'industrie de transformation depuis le début de la Révolution Industrielle -par retrait progressif de matière ou déformation de matière-. Toutes les technologies connues d'impression 3D sont basées sur la formation de l'objet virtuel 3D en lamelles successives 2D de fine épaisseur. Pour un exposé synthétique de la question, cf. [BER 14] et/ou dans une re-contextualisation économique et managériale [BOU 14].
} 
En outre, d'un certain point de vue, le bricolage et/ou l'improvisation peuvent être perçus comme une pratique participant à la réduction d'incertitude. En effet, si l'incertitude est inhérente à l'activité économique, l'organisation s'avère réductrice d'incertitude [KNI 21]. Ceci débouche d'ailleurs sur un débat théorique ontologique essentiel (entre Coase et Knight [BOU 96]) pour saisir la nature de la firme. La réduction d'incertitude est indispensable à la prise de décision et à la réalisation d'activités économiques et commerciales ; par extension, les processus participant à la dynamique organisationnelle, essentiellement d'ailleurs ceux de types informels, car souples et adaptatifs, et reposant notamment sur le bricolage et l'improvisation (et moins les procédures officielles instituées), servent à favoriser la coordination latérale et la réalisation de tâches complexes indispensables pour concevoir et innover.

Par ailleurs, la responsabilité sociétale de l'entreprise (RSoE), et du manager [BOW 53], étant au coeur désormais d'une stratégie, toute initiative ou nécessité interne allant dans ce sens doit être favorisée. Le bricolage et/ou l'improvisation constituent une double pratique utilisée en soi car indispensable dans le fonctionnement d'une organisation humaine mais aussi doublement utile car particulièrement adaptée pour une démarche compatible à la RSoE (à condition de parvenir à minimiser ou d'éliminer l'impact sur l'environnement). Dès lors, le bricolage (et/ou l'improvisation dans une moindre mesure) innovant est aussi en cohérence, voire indispensable, à une démarche conséquentiste [BOU 15b] dans la mesure où faire avec le moyens du bord, et même la rareté de ressources, pour trouver des solutions astucieuses (dans un temps imparti ou non) dénote d'abord une maîtrise respectueuse des matériaux et/ou des outils et assure ensuite l'évitement des gâchis et rebuts éventuels ainsi que, potentiellement, l'élimination de tout impact sur l'environnement. Cette prise en compte environnementale implique alors, évidemment, l'intégration du cycle de vie complet (tant des matériaux ou éléments intrants que des artefacts à réaliser) dès la conception en amont (ex ante). La démarche conséquentiste peut se définir comme la prise en compte ex ante dans la conception des conséquences prévisibles de la production et/ou de l'utilisation d'un bien : agir économiquement sans générer, consciemment ex ante, d'effets nuisibles ex post.

\section{Bibliographie}

[ABE 85] Abernathy W., Clark K., "Innovation: Mapping the Winds of Creative Destruction", Research Policy, vol.14, p.3-22, 1985.

[ALL 83] AlLEN R., "Collective Inventions", Journal of Economic Behaviour and Organization, vol.4,p.1-24, 1983.

[ALT 88] AltShULLER G., Creativity as an Exact Science, Gordon\&Breach, 1988.

[AND 12] ANDERSON C., Makers: The New Industrial Revolution, Crown Business Book, 2012.

[BAK 05] BAKER T., Nelson R., "Creating something out of nothing: Resource construction through entrepreneurial bricolage", Administrative Science Quarterly, vol.50, p.329-366, 2005.

[BAK 03] BAKER T., MINER A., EESLEY D., "Improvising firms: bricolage, retrospective interpretation \&improvisational competencies in the founding process", Research Policy, vol.32, p.255-276, 2003.

[BEN 97] BENYUS J., Biomimicry, Innovation Inspired by Nature, Harper Collins, New-York, 1997.

[BER 14] BERCHON M., "Impression 3D", Pour la Science, nº436, p.22-28, 2014.

[BOU 20] BouviER-PATRON P., "Stratégies d'Entreprise \& Innovation Frugale Environnementale : proposition d'une matrice stratégique", Recherches en Sciences de Gestion, n¹36, p.39-65, 2020.

[BOU 17a] BOUVIER-PATRON P., "Cahier des charges et relation client-fournisseurs : une tentative de synthèse", Gérer \& Comprendre, $\mathrm{n}^{\circ} 129$, Septembre 2017, p.33-43, 2017.

[BOU 17b] Bouvier-PATRON P., "Innovation Frugale Environnementale : Futur Avantage Concurrentiel de la Firme ?", Revue Recherches en Sciences de Gestion, ${ }^{\circ}{ }^{118}$, p.23-41, 2017.

[BOU 15a] Bouvier-Patron P., "FabLab et Extension de la Forme Réseau : Vers une Nouvelle Dynamique Industrielle?", Innovations, De Boeck Sup., nº4, 2015/2, p.165-188, 2015. 
[BOU 15b] Bouvier-Patron P., "Coût Global, Conséquentisme et Stratégie de Développement Durable", Management International, vol.20, $\mathrm{n}^{\circ} 1 \mathrm{p} .114-122,2015$

[BOU 14] BOUVIER-PATRON P., Économie et Management de l'Entreprise Innovante: Réseaux, Territoire et Développement Durable, collection L'Esprit Économique, L'Harmattan, 2014.

[BOU 11] BOUVIER-PATRON P., Entreprise et Innovation : Vers l'inter-organisation innovante responsable ?, collection L'esprit économique - série Economie et Innovation, L'Harmattan, 2011.

[BOU 96] BouVIER-PATRON P., "La question de la réduction d'incertitude chez F.Knight", L'Actualité Economique, n ${ }^{\circ}$, p.397-415, 1996.

[BRA 02] Braungart M., McDonough W., Cradle to Cradle: Remaking the Way We Make Things, North Point Press, New-York, 2002.

[BOW 53] BOWEN H., Social Responsibilities of the Businessman, Harper\&Brother, New York, 1953.

[BOW 95] Bower J., Christensen C., "Disruptive Technologies : Catching the Wave", Harvard Business Review, January-February, 1995.

[BRO 91] BROwN J., DUGUID P., "Organizational learning and communities of practice: towards a unified view of working, learning and innovation", Organization Science, vol.2, p.40-57, 1991.

[BRO 08] BRown T., "Design Thinking", Harvard Business Review, June, p.85-92, 2008.

[CHA 62] CHANDLER A., Strategy and Structure, Chapters in the History of the Industrial Enterprise, MIT Press, 1962.

[CHES 03] ChESBRough H., Open Innovation: The New Imperative for Creating \&Profiting from Technology, Harvard Business School Press, 2003.

[CHEV 14] CheVASSUS-AU-Louis N., Théories du complot, First Éditions, 2014.

[COH 90] Cohen W., Levinthal D., "Absorptive Capacity: A new perspective on Learning and Innovation", Administrative Science Quarterly, p.128-152, 1990.

[CUN 14] Cunha M., Rego A., Oliveira P., HabiB N., "Product Innovation in Resource-Poor Environments: Three Research Streams", Journal of Product Innovation Management, vol.31, n², p.202-210, 2014.

[GER 05] GERSHENFELD N., Fab : the coming Revolution on your Desktop-from PC to personal Fabrication, Basic Books, 2005.

[GEY 04] GeYER R., JACKSON T., "Supply Loops and Their Constraints: The Industrial Ecology of Recycling and Reuse", California Management Review, vol.46, n², Winter, p.55-73, 2004.

[HAL 12] Halme M., Lindeman S., LinNA P., "Innovation for inclusive business: Intrapreneurial bricolage in multinational corporations", Journal of Management Studies, vol.49, nº 4, p.743-84, 2012.

[HAR 84] HARTJE V., LAURIE L., Adoption rules for pollution control innovations: end-of-pipe versus integrated technologies, International Institute for Environment and Society, 1984.

[KAM 01] KAMOCHE K., CUNHA M., "Minimal structures: From jazz improvisation to product innovation", Organization Studies, vol.22, p.733-64, 2001.

[KNI 21] KNIGHT F., Risk, Uncertainty and Profit, The Riverside Press Cambridge, 1921.

[LEV 62] LeVi-Strauss C., La Pensée Sauvage, Plon, 1962.

[MIN 01] Miner A., BASSOFF P., MOORMAN C., "Organizational improvisation and learning: a field study", Administrative Science Quarterly, vol.46, p.304-337, 2001.

[MOO 98a] MOORMAN C., MiNER A., "The convergence between planning and execution: Improvisation in new product development", Journal of Marketing, vol.62, p.1-20, 1998.

[MOO 98b] MoORman C., Miner A., "Organizational Improvisation and Organizational Memory", Academy of Management Review, vol.23, nº 4, p.698-723, 1998.

[NOH 94] NOHRIA N., BERKLEY J., "Whatever happened to the take-charge manager?", Harvard Business Review, January-February, p.128-37, 1994.

[PEI] PeIRCE C., 1839-1914, Collected Papers of Charles Sanders Peirce, C.Hartshorne \& P.Weiss (eds) (vol.1-6) \& A.Burks (vol.7-8), Cambridge MA : Belknap Press, 1931-1958.

[PER 01] PERRIN J., Concevoir l'Innovation Industrielle, Édition du CNRS, 2001. 
[PRA 05] PRAHALAD C., The Fortune at the Base of the Pyramid: Eradicating Poverty through Profits, Philadelphia, PA, Wharton School Publishing, 2005.

[PSI 11] PsILlOS,S., "An Explorer upon Untrodden Ground : Peirce on Abduction", Handbook of the History of Logic, Vol.10 Inductive Logic, S.Hartmann \& J.Woods eds / General editors : D.Gabbay \& J.Woods, Elsevier, p117-151, 2011.

[RAY 99] RAYMOND E., The Cathedral and the Bazaar, O'ReillyMedia, Inc, 1999.

[SCH 42] SCHUMPETER J., Capitalism, Socialism and Democracy, Harper\& Row, 1942.

[SCH 39] SCHUMPETER J., Business Cycles, Mc Graw Hill, New York, 1939.

[SEY 07] SeYfang,G., SMITH,A., "Grassroots innovations for sustainable development : Towards a new research and policy agenda", Environmental Politics, vol.16,n²,p.584-603, 2007.

[SIM 78] SIMON H., "Rationality as Process and as Product of Thought", American Economic Review (AEA May), p.1$16,1978$.

[SIM 76] SIMON H., "From Substantive to Procedural Rationality", in Method and Appraisal in Economics, edited by S.J Latsis, p.129-148, 1976.

[SIM 69] SIMON H., The science of the artificial, MIT Press, 1969.

[VER 66] VERNON R., "International investment \& trade in the product cycle", Quarterly Journal of Economics, vol.80, $\mathrm{n}^{\circ} 2, \mathrm{p} .190-207,1966$

[VON 76] VON HIPPEL E., "The dominant Role of Users in the Scientific Instrument Innovation Process", Research Policy, n ${ }^{\circ}$, p.212-225, 1976.

[WEI 93] WEICK K., "The collapse of sensemaking in organizations: the Mann Gulch disaster", Administrative Science Quarterly, vol.38, p.628-652, 1993. 